\title{
FAM9C plays an anti-apoptotic role through activation of the PI3K/Akt pathway in human hepatocellular carcinoma
}

\author{
JUN-DONG ZHOU ${ }^{1 *}$, FEI SHEN ${ }^{2 *}$, JIAN-SONG JI $^{3 *}$, KAI ZHENG $^{1}$, MIN HUANG $^{1}$ and JIN-CHANG WU ${ }^{1}$ \\ ${ }^{1}$ The Core Laboratory of the Suzhou Cancer Center and Department of Radiotherapy of the Suzhou Hospital Affiliated to \\ Nanjing Medical University, Suzhou 215001; ${ }^{2}$ The Key Laboratory of Thrombosis and Hemostasis of Ministry \\ of Health, Jiangsu Institute of Hematology, The First Affiliated Hospital of Soochow University, \\ Suzhou 215001; ${ }^{3}$ Lishui Center Hospital, Lishui, Zhejiang 32300, P.R. China
}

Received April 25, 2013; Accepted June 7, 2013

DOI: $10.3892 /$ or.2013.2592

\begin{abstract}
The function of FAM9C encoding a testis-exclusively expressed and nuclear-localized protein remains unknown. In the present study, we evaluated the role of FAM9C in human hepatocellular carcinoma. We found that among three FAM9 family members, only FAM9C was frequently upregulated in HCC specimens compared with that in corresponding adjacent non-cancer liver tissues. FAM9C was located in the nucleus of HCC cells, as shown by both western blotting and immumofluorescence assays. Significantly, FAM9C overexpression promoted proliferation, clonogenicity in an anchorage-dependent manner, in vivo tumorigenicity of YY-8103, and Huh-7 cells. In contrast, FAM9C knockdown suppressed proliferation, anchorage-dependent colony formation and in vivo tumorigenicity of QGY-7703, and BEL-7404 cells. However, FAM9C had no significant effects on cell cycle progression when FAM9C was stably overexpressed in Huh-7 cells or knocked down in BEL-7404 cells. Most importantly, FAM9C regulated activation of Akt and UV-induced apoptosis in $\mathrm{HCC}$ cells. FAM9C overexpression increased the phosphorylation levels of Akt and anti-apoptotic ability of Huh-7 cells, whereas endogenous $F A M 9 C$ knockdown reduced the phosphorylated levels of Akt and anti-apoptotic ability of BEL-7404 cells. Furthermore, the anti-apoptotic function of FAM9C could be prevented when the PI3K-Akt pathway was in a loss-offunction caused by RNA interference against Akt or PI3K
\end{abstract}

Correspondence to: Dr Jin-Chang Wu or Dr Min Huang, The Core Laboratory of the Suzhou Cancer Center and Department of Radiotherapy of the Suzhou Hospital Affiliated to Nanjing Medical University, Suzhou 215001, P.R. China

E-mail: wjinchang@sina.com

E-mai: szhuangmin@163.com

\section{* Contributed equally}

Key words: hepatocellular carcinoma, FAM9C, cancer testis, PI3K-Akt, anti-apoptosis, RNA interference inhibitor LY294002 in HCC cells. Taken together, our data demonstrate that FAM9C as a novel cancer testis gene plays an anti-apoptotic role in human hepatocellular carcinoma through activating the PI3K/Akt signaling pathway, and serves as a promising target for HCC therapy.

\section{Introduction}

Hepatocellular carcinoma (HCC) is the most common primary hepatic malignancy with the continually increasing incidence, and the second major cause of cancer-related deaths worldwide $(1,2)$. In China, the most common cause of this neoplasm is the chronic infection induced by the hepatitis B virus. Other important etiological factor are cirrhosis, chronic viral hepatitis (hepatitis $\mathrm{C}$ virus), alcohol abuse, obesity, hemochromatosis, $\alpha 1$-antitripsin deficiency and toxins similar to aflatoxin (3). Because recent development of genomic technologies has empowered survey of molecular aberrations and deregulations directly from patient specimens in a comprehensive way, some presented prognostic/predictive targets for $\mathrm{HCC}$ biomarkers improved the prognosis of patients by guiding treatment decision and allocation of medical resources (4-6). Cancer/testis genes are normally expressed by gametes and trophoblasts, as well as aberrantly expressed in a range of malignant tumors of unrelated histological origin, including the liver. CT genes are a class of closely cancer-related biomarkers. Since germline stem cells and their trophoblastic derivatives share many characteristics with tumour cells, the activation of portions of the germline gene-expression in cancer cells could contribute to the malignant phenotype, including proliferation, survival, invasiveness, immune evasion and metastatic capacity (7).

A number of CT genes have been found expressed in HCC and their products are promising targets for antigen-specific immunotherapy of this tumor. For example, the mRNA expressions of MAGE1, SSX-1, CTp11 and HCA587, were detectable in diverse percentage of HCC samples examined $(8,9)$. The brother of the regulator of imprinted sites (BORIS) as a novel member of the CT antigens may be an auxiliary diagnosis index and a novel favorable prognostic indicator of HCC (10). However, whether their recurrent expression contributes to tumorigenesis, is still under investigation. Recent studies 
demonstrate the important role of CT genes in malignant features of HCC cells. A known member of CT antigens family, NY-ESO-1, whose expression is associated with worse HCC outcome, increases tumor cell migration (11).

To identify novel CT genes, some genes with testisexclusive expression have been searched for through published literature. In the present study, we paid attention to a reported gene family, which included three members with testis-exclusively expression, FAM9A, FAM9B and FAM9C (12) and investigated their expression profiles in human hepatocellular carcinoma (HCC). Interestingly, among the three members, only FMA9C was frequently upregulated in HCC specimens compared with that of the corresponding non-cancerous livers. Nevertheless, the physiological and the pathological function of FAM9C is not understood. Therefore, we further evaluated the role of FAM9C in HCC cells. Significantly, the resulting data demonstrated that FAM9C overexpression promoted proliferation, colony-forming growth and in vivo tumorigenicity of HCC cells, and RNA interference against FAM9C suppressed these phenotypes in HCC cells. We found that FAM9C endowed HCC cells with resistance to apoptosis through PI3K-Akt pathway. Our results reveal that upregulated FAM9C as a novel cancer testis gene plays an anti-apoptotic role in human HCC.

\section{Materials and methods}

Tissue specimens and cell lines. HCC specimens used in the present study were obtained from HCC patients who underwent liver resection and gave their informed consent. The following liver tumor-derived cell lines SSMC-7721, QGY-7703, BEL-7404, BEL-7405, YY-8103 and Huh-7, were employed in the present study. These cell lines were grown in Dulbecco's modified Eagle's medium (DMEM), supplemented with heatinactivated $10 \%$ fetal bovine serum (Gibco, Carlsbad, CA, USA) at $37^{\circ} \mathrm{C}$ in a $5 \% \mathrm{CO}_{2}$ humidified incubator.

Antibodies and reagents. Antibodies against FAM9C, GAPDH, Lamin $A$ and $\beta$-actin were from Santa Cruz Biotechnology Inc. (Santa Cruz, CA, USA). Antibodies against phospho-Akt (pAkt and Ser473) and Akt were from Cell Signaling Technology (Beverly, MA, USA). Propidium iodide (PI), 3-(4,5-dimethylthiazol-2-yl)-2,5-diphenyltetrazolium bromide (MTT), 5-Aza-2'-deoxycytidine (5-Aza-dC), 4'-6-diamidino-2-phenylindole (DAPI) and PI3K inhibitor LY294002 were purchased from Sigma Chemical Co. (St. Louis, MO, USA).

5-Aza-2'-deoxycytidine (5-Aza-dC) treatment. SSMC-7721 and BEL-7405 cells were seeded at a density of $5 \times 10^{5} / 100-\mathrm{mm}$ dishes, cultured for $48 \mathrm{~h}$, and treated with $2 \mathrm{mM} \mathrm{5-Aza-dC}$ (Sigma Chemical), a demethylating agent. Forty-eight hours after treatment, cells are washed with PBS and fresh medium was added. Cells are further incubated for another $48 \mathrm{~h}$ before isolation of total cellular RNA.

RNA extraction, reverse transcription-PCR and real-time $P C R$. RNA from HCC specimens and cell lines were extracted using TRIzol ${ }^{\circledR}$ Reagent (Invitrogen Life Technologies,
Carlsbad, CA, USA) according to the manufacturer's instructions. Total RNA was treated with DNase I to avoid DNA contamination, the quality and quantity of extracted RNA was confirmed by NanoDrop 2000 (Thermo Fisher Scientific, Waltham, MA, USA). RNA reverse transcription reaction was performed using M-MLV reverse transcriptase (Promega), according to the manufacturer's instructions. The primers sequences used in this study were as follows: upstream: 5'-TAG GAGCTGGAGGCTGAGAG-3' and downstream: 5'-GGTTG AAGAGACAATGAAGTGCC-3' for FAM9A gene amplification (NM_174951.2); upstream: 5'-GCAGTGCAGTGGTG TGATCT-3' and downstream: 5'-CTTTCCTGCATGCTTCT TCC-3' for FAM9B gene amplification (NM_205849.1); upstream: 5'-CTGAGGGAGCAACATCAGGG-3' and downstream: 5'-TTGGTATCAACCCCCGTGTG-3' for FAM9C gene amplification (NM_174901.4); and $\beta$-actin, a typical housekeeping gene used as the internal control, upstream: 5'-AATCGTGCGTGACATTAAGGAG-3' and downstream: 5'-ACTGTGTTGGCGTACAGGTCTT-3'. All reactions were performed in triplicate in an Applied Biosystems 7300 Real-time PCR system. The relative mRNA level of the target gene was calculated using the comparative threshold cycle method $\left(2^{-\Delta \Delta C t}\right)$ normalized by $\beta$-actin expression (13).

Immunohistochemistry and immunofluorescence assay. Formalin-fixed HCC samples were paraffin-embedded and cut into $4-\mu \mathrm{m}$ sections. The sections were deparaffinized and dehydrated, and then treated with methanol containing $0.3 \% \mathrm{H}_{2} \mathrm{O}_{2}$ to inhibit endogenous peroxidase. The slides were incubated with anti-FAM9C goat polyclonal antibody (Santa Cruz Biotechnology) at $37^{\circ} \mathrm{C}$ for $2 \mathrm{~h}$ and then at $4^{\circ} \mathrm{C}$ overnight, followed by incubation with a horseradish peroxidase-conjugated anti-goat antibody (Dako Japan Ltd., Kyoto, Japan) at $37^{\circ} \mathrm{C}$ for $1 \mathrm{~h}$. The signals were detected using Diaminobenzidine Substrate kit (Vector Laboratories, Burlingame, CA, USA). Counterstaining was performed with hematoxylin. In addition, the slides with HCC specimens and the corresponding adjacent non-HCC livers were simultaneously used in the immunohistochemistry staining, and were then assessed by visual inspection and the estimation of the percentage of immunopositive cells. Immunofluorescence assay was performed to detect endogenous FAM9C in BEL-740 4 cells. These cells were plated on polylysine-treated slides and then incubated at $37^{\circ} \mathrm{C}$ for $1 \mathrm{~h}$. The fixed cells were blocked with phosphate-buffered saline buffer containing $5 \%$ bovine serum albumin and then stained with anti-FAM9C antibody (Santa Cruz Biotechnology) at $4^{\circ} \mathrm{C}$ overnight, followed by incubation with fluorescein isothiocyanate-conjugated goat anti-IgG antibody (Gibco-BRL, Grand Island, NY, USA) at $4^{\circ} \mathrm{C}$ for $2 \mathrm{~h}$. After rinsing, the slides were analyzed using immunofluorescence microscopy.

RNA interference (RNAi). Small interference RNAs (siRNAs) targeting FAM9C, Akt and negative control siRNA (si-NC) were chemically synthesized (Invitrogen-Life Technologies). Two siRNAs against FAM9C with high efficiency were used in the functional experiment assays, and their sequences were as follows: FAM9C-si1, sense, 5'-GGACCAGUUGGAGGUU CAAdTdT-3', antisense, 5'-UUGAACCUCCAACUGGUCC dTdT-3'; FAM9C-si2, sense, 5'-CCAAGGAGCUAGAAGAU 
AUdTdT-3', antisense, 5'-AUAUCUUCUAGCUCCUUGT dT-3'; si-NC, sense, 5'-UUCUCCGAACGUGUCACGUdT dT-3', antisense, 5'-ACGUGACACGUUCGGAGAAdTdT-3'. As well, the sequences of a siRNA against Akt were as follows: sense, 5'-GCACCUUCAUUGGCUACAAdTdT-3', antisense, 5'-UUGUAGCCAAUGAAGGUGCdTdT-3'.

Construction of recombinant plasmids. The full-length FAM9C ORF (501 bp, GenBank accession number NM_174901.4) was amplified by RT-PCR from BEL-7404 cells cDNA. The primers for FAM9C ORF were as follows: forward, 5'-TCAGAATTCATGGCTGCCAAGGACCAGT TGGAGG-3'; reverse, 5'-TCAGGTACCTCAATCTCTTTCA GTTCCTTTCCCA-3'. The PCR product was then inserted into pcDNATM3.1B (Invitrogen) through $E c o$ RI- and $K p n I-$ adapter. In addition, synthesized DNA nucleotide fragments of short hairpin RNA (shRNA) for knocking down endogenous FAM9C, was inserted into pSUPER (Oligoengine; Seattle, WA, USA). The sequences of these synthesized oligonucleotides for FAM9C silencing were as follows: forward, 5'-GAT CCCCGGACCAGTTGGAGGTTCAATTCAAGAGATTGA ACCTCCAACTGGTCCTTTTTGGAAA-3', reverse, 5'-AGCT TTTCCAAAAAGGACCAGTTGGAGGTTCAATCTCTTGA ATTGAACCTCCAACTGGTCCGGG-3' (FAM9C-sh1); forward, 5'-GATCCCCCCAAGGAGCTAGAAGATATTTC AAGAGAATATCTTCTAGCTCCTTGGTTTTTGGAAA-3', reverse, 5'-AGCTTTTCCAAAAACCAAGGAGCTAGAAG ATATTCTCTTGAAATATCTTCTAGCTCCTTGGGGG-3' (FAM9C-sh2); pSUPER-shNC with irrelevant nucleotide sequence serves as a negative control, and sequences of shNC were as follows: forward, 5'-GATCCCCTTCTCCGAACGT GTCACGTTTCAAGAGAACGTGACACGTTCGGAGAATT TTTGGAAA-3', reverse, 5'-AGCTTTTCCAAAAATTCTCCG AACGTGTCACGTTCTCTTGAAACGTGACACGTTCGGA GAAGGG-3'.

Cell transfection. Cell transfection was performed by Lipofectamine 2000 (Invitrogen) according to the manufacturer's instructions.

Cell growth curve. HCC cells with FAM9C overexpression or knockdown were seeded in triplicate into 96-well plates $(3,000$ cells/well) respectively and then incubated with $5 \mathrm{mg} / \mathrm{ml}$ (10 $\mu \mathrm{l}) \mathrm{MTT}$ for $4 \mathrm{~h}$ at $37^{\circ} \mathrm{C}$, then the medium was replaced with $100 \mu$ l dimethylsulfoxide (DMSO) for 10 min every 24-h intervals for 5-6 days. Cell growth curves were generated by calculating the mean value of the optical density measurements at $492 \mathrm{~nm}$ using a microplate reader. All experiments were independently repeated at least 3 times.

Colony formation assay. FAM9C was overexpressed in HCC cell lines Huh-7 and YY-8103 through the transfection with recombinant pcDNA3.1-FAM9C plasmid, additionally endogenous FAM9C was knocked down in QGY-7703, BEL-7404 cells via pSUPER-shFAM9C plasmids. Transfected cells were cultured on 100-mm dishes for colony formation, followed by the addition of G418 (Life Technologies) to the medium at a final concentration of $0.6-1 \mathrm{mg} / \mathrm{ml}$. After 3-4 weeks, the remaining colonies were washed twice with PBS and counted on crystal violet-stained dishes. All experiments for observing colony formation were independently repeated at least three times.

Establishment of stable cell lines. To establish a stably FAM9C-expressing HCC cell line, HuH-7 cells were transfected with the recombinant plasmid pcDNA3.1B-FMA9C, then cultured by G418 (Life Technologies). An immunoblot assay was employed to examine the expression of FAM9C in nine individual colonies. One colony with steady and strong FAM9C expression was selected as study object, and another colony without ectopic FAM9C expression was used as mock object. In addition, BEL-7404 cell line with stable FAM9C knockdown was also established by transfection with plasmid pSUPER-FAM9C-sh2, with the methods mentioned above. A colony of BEL-7404 cells transfected with plasmid pSUPERshNC containing irrelevant sequence was used as a negative control.

Western blot assay. Cultured HCC cells were harvested with trypsin-EDTA and then centrifuged at $500 \mathrm{x}$ g for $5 \mathrm{~min}$. Cytoplasmic and nuclear proteins were extracted using NE-PER ${ }^{\circledR}$ Nuclear and Cytoplasmic Extraction reagents (Thermo Scientific Pierce), according to the manufacturer's instructions. Protein extracts were separated by $12 \%$ SDS-PAGE and transferred onto Hybond-C nitrocellulose membranes (Amersham Life Science, Buckinghamshire, UK). After blocking with PBS containing 5\% non-fat milk, the membrane was incubated for immunoblot analysis with primary antibodies, followed by incubation with an IRDye 800DX-conjugated, affinity-purified secondary antibody. Signals were detected using the Odyssey Infrared Imaging system (LI-COR Biosciences).

Flow cytometric analysis. Flow cytometry was used to analyze cell cycle and cell apoptosis. For DNA content analysis, cells were fixed in $70 \%$ ethanol, rehydrated in PBS, and treated for 30 min with RNase A $(10 \mathrm{mg} / \mathrm{ml})$ and for 5 min with propidium iodide $(10 \mu \mathrm{g} / \mathrm{ml})$. For UV-induced apoptosis analysis, $3 \mathrm{~J} / \mathrm{cm}^{2}, 5 \mathrm{~min}$ UV-irradiated cells $\left(1 \times 10^{6}\right.$ cells $)$ were harvested and washed twice with cold PBS. Then, Annexin V-FITC staining was performed according to the protocol provided by the manufacturer (BD Biosciences Pharmingen, San Diego, CA, USA). Briefly, cells were resuspended in binding buffer at a concentration of $1 \times 10^{6}$ cells $/ \mathrm{ml}$, and $5 \mu \mathrm{l}$ of Annexin-FITC and $100 \mu \mathrm{l}$ cell suspension ( $1 \times 10^{5}$ cells) were gently mixed and incubated for $15 \mathrm{~min}$ at room temperature in the dark. Cells were analyzed for apoptosis by flow cytometry on a FACSCalibur (Becton-Dickinson, Mountain View, CA, USA).

Tumorigenicity assay in nude mice. A total of $2 \times 10^{6}$ offspring Huh-7 cells stably expressing ectopic FAM9C were subcutaneously injected into the flank of nude mice, and the same amount of progeny Huh-7 cells without ectopic FAM9C expression as mock control were injected into the opposite flank of the same mice. Cells $\left(2 \times 10^{6}\right)$ derived from BEL-7404 with stable knockdown of endogenous FAM9C were injected subcutaneously into the flank of nude mice, and the same amount of BEL-7404 cells from the colony transfected with pSUPER-shNC were injected into the opposite flank of the same mice. Tumor growth kinetics was estimated by measuring tumor size and 
A
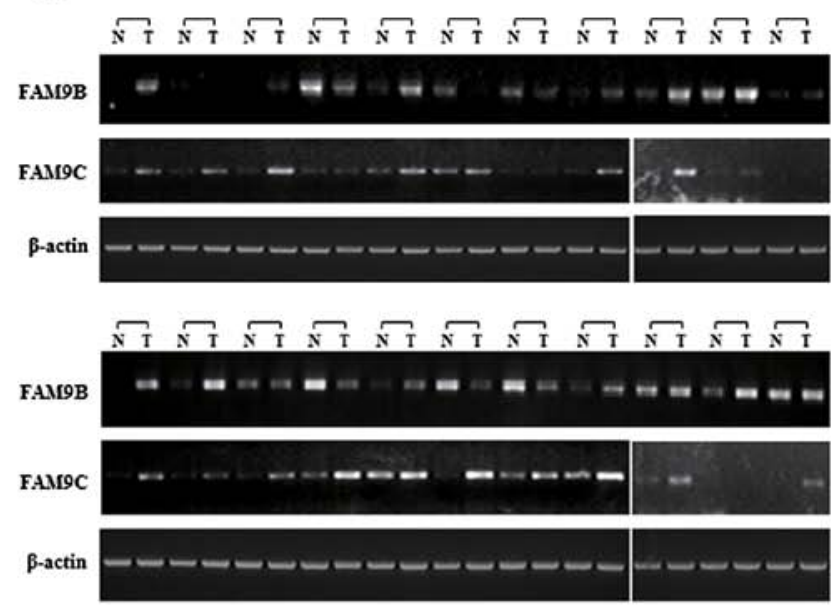

C

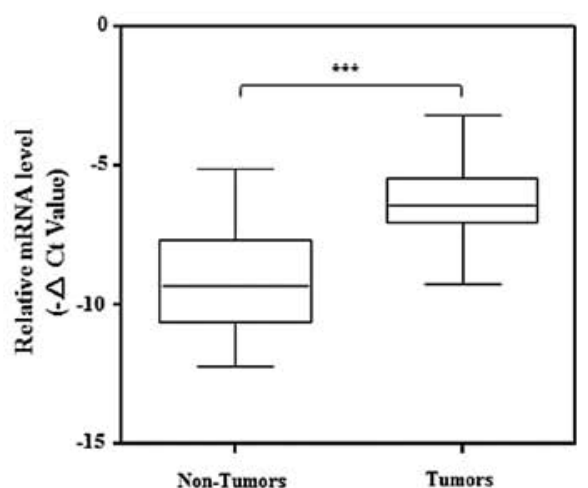

E

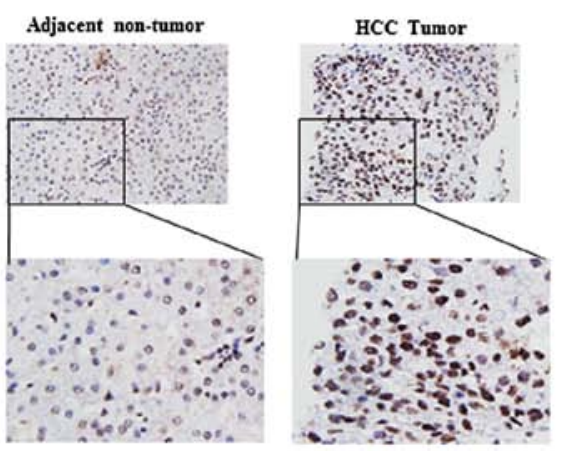

B
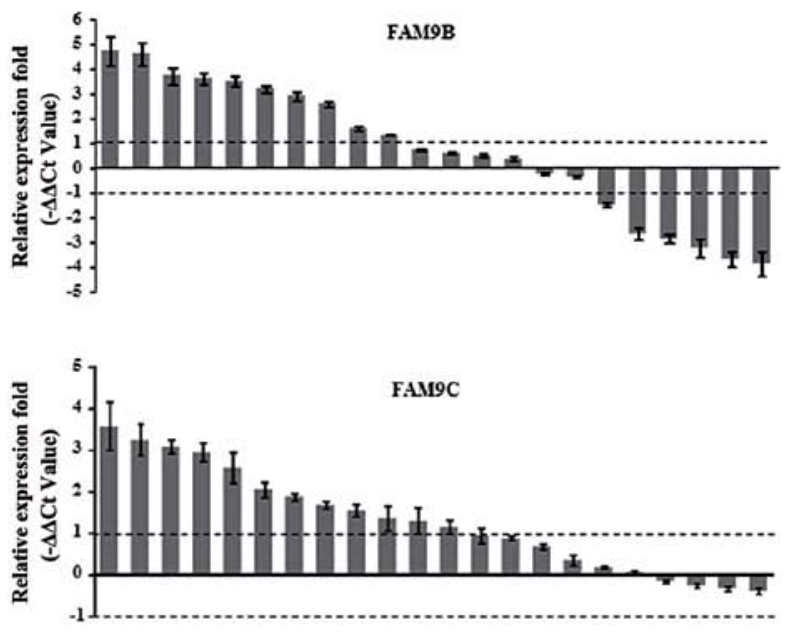

D
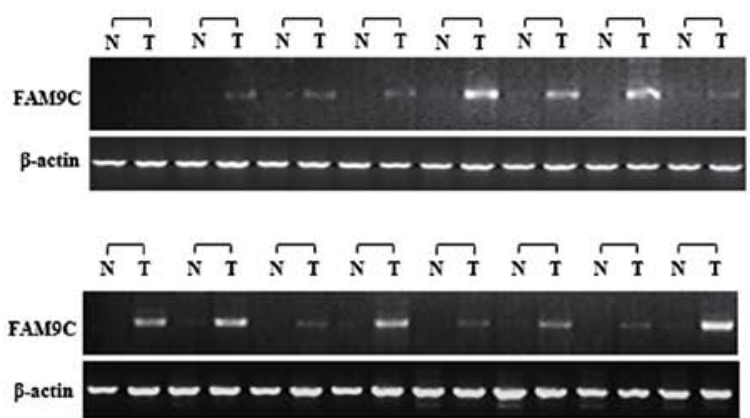

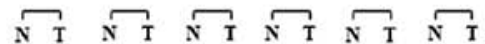
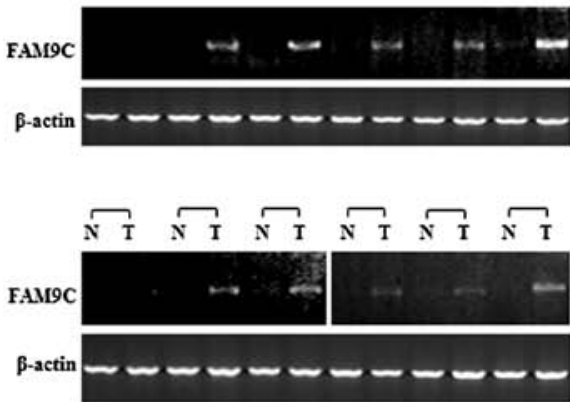

Figure 1. Expression profile of FAM9C in HCC specimens. (A and B) RT-PCR was performed to investigate the expression level of three gene members of FAM9 family in $22 \mathrm{HCC}$ tumor tissues (T) and their matched adjacent non-cancerous livers (N), where $\beta$-actin was used as an internal control. Except for FAM9A, these specific PCR products of FAM9B and FAM9C were subject to (A) agarose gel electrophoresis and (B) real-time RT-PCR analysis. The columns show the relative expression fold-change $\left({ }^{-\Delta \Delta C t}\right.$ Value $)$ of mRNA levels of FAM9B, FAM9C in HCC specimens. Data are presented as the means \pm SD of three independent experiments. (C) Real-time RT-PCR analysis of FAM9C was carried out on additional 46 paired HCCs and adjacent non-cancerous livers. For each sample, the relative mRNA level of FAM9C was normalized based on that of $\beta$-actin. The line within each box represents the median $-\Delta C t$ value; the upper and lower edges of each box represent the 75th and 25th percentile, respectively; the upper and lower bars indicate the highest and lowest values determined, respectively. Statistical analysis was performed by a paired two-tailed t-test. ${ }^{* * * *} \mathrm{P}<0.001$. (D) The elevated mRNA expression of FAM9C was typically showed when the addition 46 HCC specimens and corresponding adjacent non-cancerous livers were examined by RT-PCR, where $\beta$-actin was used as an internal reference. (E) Representative images of the immunohistochemical staining of a paired HCC specimen with the antibody against FAM9C are shown.

volume every 3-4 days. Tumor volume was determined using the following formula: $1 / 2 \mathrm{x}$ width $^{2} \mathrm{x}$ length.

Statistical analysis. Statistical analysis was performed by the Student's t-test using SSPS software (SPSS, Inc., Chicago, IL, USA). P-value $<0.05$ was considered to indicate a statistically significant result.

\section{Results}

FAM9C was frequently upregulated in human HCC. To evaluate the role of FAM9 members in HCC, we first investigated the transcriptional levels of three FAM9 family members, FAM9A, FAM9B and FAM9C, in 22 pairs of human HCC specimens by semi-quantitative and real-time RT-PCR. The 
A



C

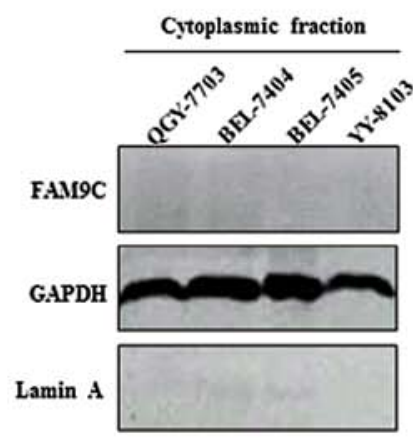

B

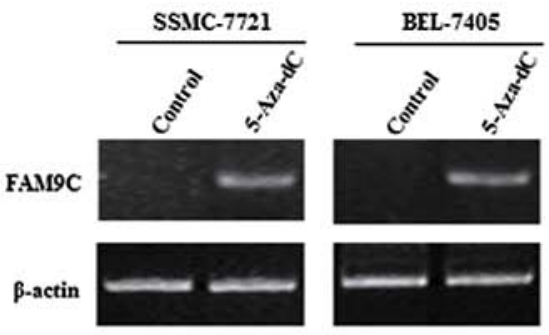

D

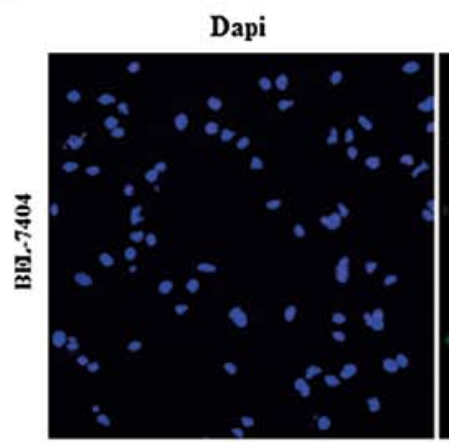

FAM9C

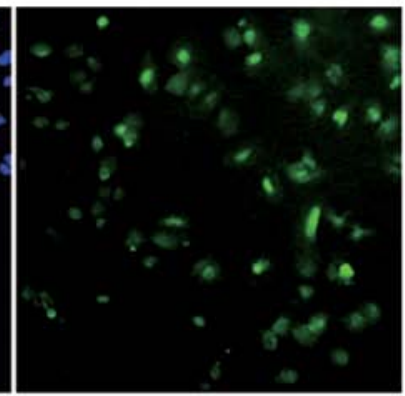

Figure 2. The expression and subcellular location of FAM9C in HCC cell lines. (A) The mRNA expression of FAM9C in six HCC cell lines was evaluated by RT-PCR. (B) SSMC-7721 and BEL-7405 cells without endogenous FAM9C expression were treated with 5-Aza-dC. The expression of FAM9C was then examined by RT-PCR and the untreated HCC cells were employed as controls. (C) Western blot assay was performed to evaluate the FAM9C expression in the cytoplasmic and nuclear extracts of four HCC cell lines, where GAPDH was used as the indicator of cytoplasmic fraction and Lamin A as the indicator of nuclear fraction. (D) Immunofluorescence assay indicated that FAM9C was located within nuclei in BEL-7404 cells. The nuclei were stained with 4',6-diamidino-2-phenylindole (DAPI).

data showed that only FAM9C mRNA was obviously elevated in more than a half of HCC specimens examined, as compared with that of corresponding non-cancerous livers, while FAM9A mRNA was not specifically amplified and FAM9B exhibited disordered expression in these specimens (Fig. 1A and B). To confirm this result, FAM9C was further evaluated in additional 46 paired human HCC specimens by semi-quantitative and real-time PCR. The result showed the relative mRNA level of FAM9C was significantly upregulated in HCC tumor specimens compared with corresponding adjacent non-tumor livers $(\mathrm{P}<0.001)$ (Fig. 1C), and the upregulation of FAM9C mRNA was obvious in 25 of the 46 HCC specimens as shown by semi-quantitative PCR (Fig. 1D). Furthermore, we also examined the expression and location of FAM9C protein in a pair of HCC specimens using immunohistochemical staining with a specific antibody against FAM9C. The resulting data showed that FAM9C protein was located in the nuclei, and presented more expression in tumor tissue than adjacent nontumor sample (Fig. 1E). Collectively, these data demonstrated that FAM9C was frequently elevated in human HCCs and as a novel cancer testis gene.

The expression and subcellular location of FAM9C in HCC cell lines. The expression pattern of FAM9C was further evaluated in some HCC cell lines by RT-PCR. The result showed that FAM9C presented relatively high expression in QGY-7703 and BEL-7404 cells but low or no expression in other cell lines examined (Fig. 2A). To investigate whether DNA methylation regulates the FAM9C expression in $\mathrm{HCC}$ cells, we treated two HCC cell lines SSMC-7721 and BEL-7405 with 5-Aza-dC, a demethylating agent. RT-PCR analysis showed that FAM9C expression was upregulated in these treated cells as compared with untreated controls (Fig. 2B). In addition, we determined the expression and subcellular location of FAM9C protein in HCC cells by a western blot assay of cytoplasmic and nuclear extracts, as well as a subcellular immunofluorescence assay. In conformity with the mRNA expression levels of FAM9C, FAM9C protein exhibited also relatively high expression in nuclear extracts of QGY-7703 and BEL-7404 cells, but without expression in cytoplasmic extracts of these cell lines (Fig. 2C). FAM9C was located in the nucleus in BEL-7404 cells, as indicated by immunofluorescence assay (Fig. 2D).

FAM9C overexpression promotes growth and colony formation of HCC cells in vitro. To evaluate the role of FAM9C on HCC cells, recombinant pcDNA3.1-FAM9C was transiently transfected into Huh-7 and YY-8103 cells with little FAM9C expression. The result showed that overexpressed FAM9C, as demonstrated by western blot assay, significantly promoted cell growth of the two HCC cell lines compared with those transfected with empty vector (Fig. 3A and B). Furthermore, FAM9C also promoted the anchorage-dependent colony formation of Huh-7 and YY-8103 cells (Fig. 3C and D). These data suggest that FAM9C overexpression plays an important role in promoting HCC cell growth, colony formation in vitro.

FAM9C knockdown inhibits cell proliferation and colony formation of HCC cells in vitro. To further investigate the effect of FAM9C on HCC cell proliferation and colony formation, we used chemically synthesized siRNAs and 
A

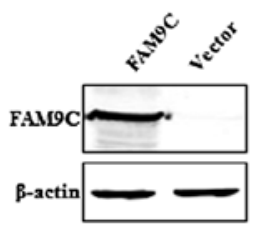

B

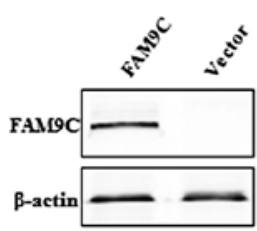

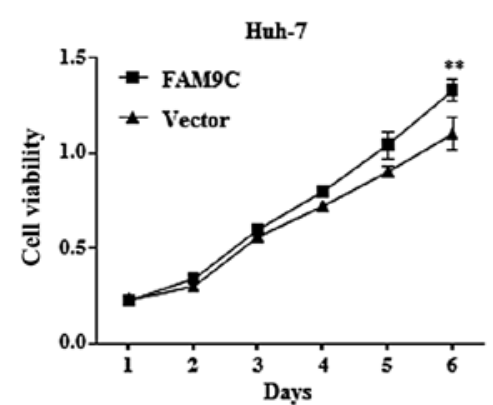

C
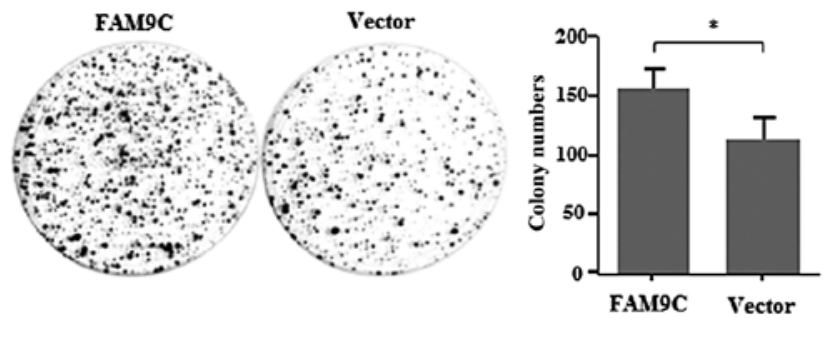

D
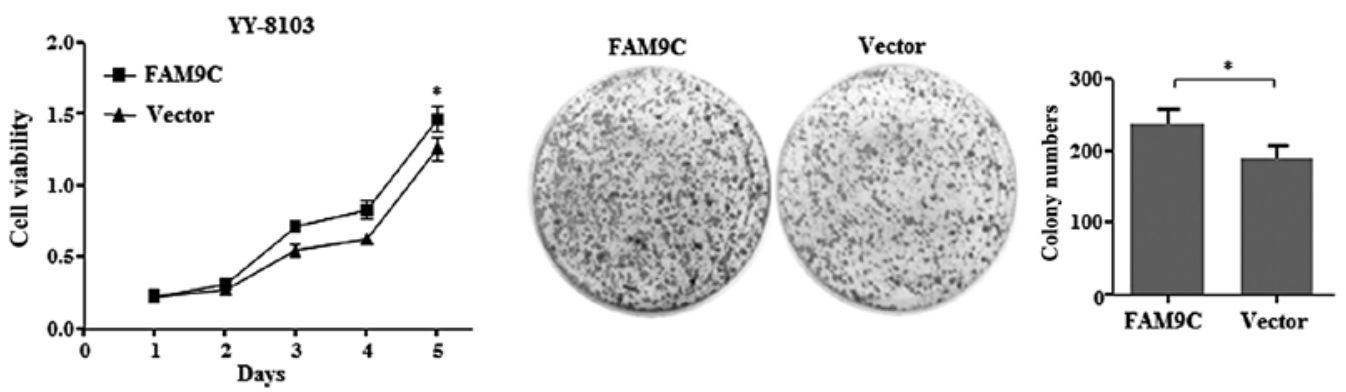

Figure 3. The effect of FAM9C overexpression on growth curve and colony formation of HCC cells in vitro. (A and B) Enforced FAM9C expression promoted the proliferation of Huh-7 (A) and YY-8103 (B) cells, where cells were transfected with pcDNA3.1B-FAM9C and the empty vector pcDNA3.1B as controls. FAM9C expression was evaluated by western blot assay (left). The experiments were repeated at least three times and the curves showed the average values of triplicate wells, with standard deviation (SD) included for each mean value (right). (C and D) Overexpressed FAM9C enhanced anchor-dependent colony formation of Huh-7 (C) and YY-8103 (D) cells, as shown by representative dishes (left) of colony formation of those transfected with pcDNA3.1B-FAM9C and pcDNA3.1B used as a control. The histogram (right) represents the relative numbers of colonies from three independent experiments, where the columns represent the average values, with $\mathrm{SD}$ (means $\pm \mathrm{SD}$ ). Statistical analysis was performed by a two-tailed t-test. ${ }^{*} \mathrm{P}<0.05,{ }^{* *} \mathrm{P}<0.01$.

shRNAs derived from recombinant pSUPER to knock down endogenous FAM9C in BEL-7404 and QGY-7703 cells. Through evaluating the knockdown efficiency of four siRNAs against FAM9C (data not shown), the two efficient siRNAs (FAM9C-si1 and FAM9C-si2) were used to silence endogenous FAM9C in BEL-7404 and QGY-7703 cells (Fig. 4A). The growth curve results showed that FAM9C knockdown significantly inhibited the proliferation of BEL-7404 and QGY-7703 cells (Fig. 4B). Moreover, pSUPER vectors carrying shRNAs (FAM9C-sh1 and FAM9C-sh2) against FAM9C were employed to FAM9C knockdown, where endogenous FAM9C was silenced in the above two HCC cell lines respectively, as demonstrated by western blot assay (Fig. 4C). The colony formation assay demonstrated that FAM9C knockdown significantly suppressed the anchorage-dependent clonogenicity of BEL-7404 and QGY-7703 cells (Fig. 4D). These collective data implied that endogenous FAM9C could be essential for maintaining the proliferation and anchorage-dependent colony formation of HCC cells in vitro.

The effect of FAM9C on the tumorigenicity of HCC cells in nude mice. To determine whether the dysregulated FAM9C contributes to hepatocarcinogenesis in vivo, Huh-7 cells $\left(2 \times 10^{6}\right)$ stably expressing FAM9C were subcutaneously injected into a flank of athymic nude mice, and the same amount of subcloned Huh-7 cells transfected with pcDNA3.1FAM9C but without ectopic FAM9C expression as Mock control were subcutaneously injected into the other flank of the same nude mice. As expected, the stably FAM9Cexpressing Huh-7 cells formed tumors faster than the mock cells without FAM9C expression under the observation for four weeks $(\mathrm{P}<0.05)$ (Fig. 5A). The removed xenograft tumors from nude mice demonstrated that FAM9C overexpression significantly promoted the tumor size and weight as compared with that of mock objects $(\mathrm{P}<0.05)$ (Fig. 5B and $\mathrm{C}$ ). These data indicated that FAM9C overexpression significantly promoted tumorigenicity in vivo of Huh-7 cells. Moreover, a total of $2 \times 10^{6}$ BEL-7404 cells, whose endogenous FAM9C was stably knocked down by plasmid pSUPER-FAM9C-sh2, were subcutaneously injected into a flank of athymic nude mice, and the same amount of cells transfected with control plasmid (pSUPER-shNC) as negative control was injected into the opposite flank of the same mice. Notably, FAM9C knockdown led to significant tumor growth retardation under observation for four weeks $(\mathrm{P}<0.01)$ (Fig. 5D), where BEL-7404 cells with FAM9C silence had low tumorigenicity in vivo in four of six mice tested, whereas cells used as controls formed tumors in all six mice (Fig. 5E). The removed xenograft tumors from nude mice showed that FAM9C silence significantly restrained the tumor size and weight as compared with that of controls $(\mathrm{P}<0.01)($ Fig. $5 \mathrm{~F})$. The result indicated that silencing of FAM9C significantly inhibited tumorigenicity in vivo of BEL-7404 cells.

FAM9C plays an anti-apoptotic role through activating the PI3K-Akt signaling pathway in HCC cells. To explore the molecular mechanisms by which FAM9C contributes to HCC cell proliferation, clonogenicity in vitro and tumorigenicity in vivo, we first analyzed the cell cycle of $\mathrm{HCC}$ cells with FAM9C overexpression or knockdown. However, FAM9C 
A

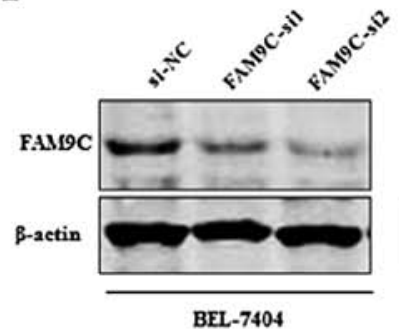

BEL-7404

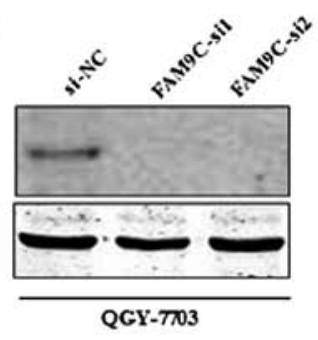

C

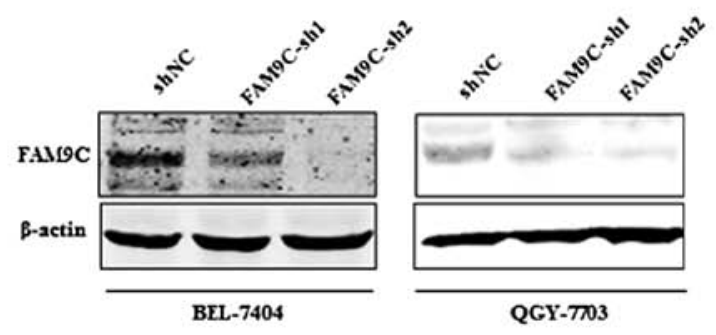

B

D
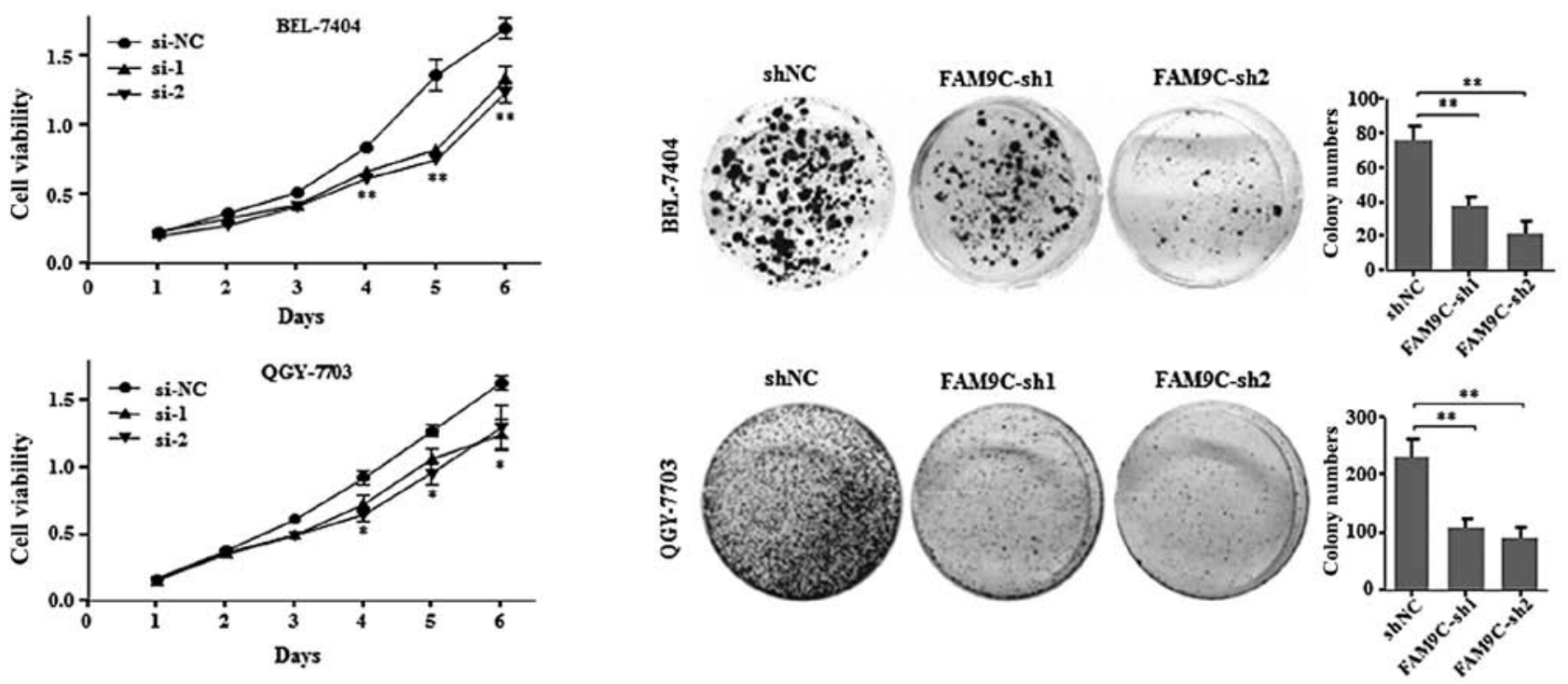

Figure 4. The effect of FAM9C knockdown on proliferation and colony formation of HCC cells in vitro. (A) The efficiency of two siRNAs (si-1 and si-2) against FAM9C was evaluated by western blot assay in BEL-7404 and QGY-7703 cells, where si-NC with irrelevant sequence was used as reference and $\beta$-actin as loading control. (B) Cell growth curves of BEL-7404 and QGY-7703 cells showed that cell proliferation was significantly inhibited by both si-1 and si-2 against FAM9C. The results represented the average value of triplicate wells, with standard deviation. (C) The knockdown efficiency of two shRNAs (sh-1 and sh-2) against endogenous FAM9C was evaluated by western blot assay in BEL-7404 and QGY-7703 cells, where $\beta$-actin was used as loading control. (D) FAM9C knockdown inhibited anchorage-dependent colony formation of BEL-7404 and QGY-7703 cells. The experiments were repeated at least three times, and the histograms represent the mean numbers of colonies from triplicate tests (means $\pm \mathrm{SD})$. Statistical analysis was performed by a two-tailed t-test. ${ }^{*} \mathrm{P}<0.05$, ${ }^{* *} \mathrm{P}<0.01$.

overexpression and knockdown did not alter cell cycle distribution of Huh-7 and BEL-7404 cells respectively (Fig. 6A and B). As reported, FAM9C protein exhibited similarity to SYCP3, a component of the synaptonemal complex (12). More importantly, SYCP3 led to activation of Akt, resulting in the upregulation of anti-apoptotic proteins (14). To determine whether FAM9C as a homologous gene of SYCP3 activates Akt signaling pathway, we examined the activity of Akt pathway by western blot assay while FAM9C overexpression or knockdown in HCC cells. We found that the phosphorylation levels of Akt significantly increased in Huh-7 cells expressing FAM9C, whereas the phosphorylation levels of Akt obviously decreased in BEL-7404 cells with FAM9C knockdown (Fig. 6C). These data indicated that FAM9C regulated activation of Akt.

To further address the role of FAM9C-induced activation of Akt in HCC cells, we investigated cell apoptosis of UV-irradiated HCC cells with FAM9C overexpression or knockdown by flow cytometry method. The resulting data demonstrated that FAM9C overexpression significantly reduced the proportion of apoptotic Huh-7 cells $(\mathrm{P}<0.01)$, while FAM9C knockdown significantly increased the apoptotic percentage of BEL-7404 cells $(\mathrm{P}<0.01)$, indicating that FAM9C endowed HCC cells with resistance to apoptosis (Fig. 6D and E). In addition, to determine whether the activation of PI3K-Akt pathway is directly responsible for the anti-apoptotic role of FAM9C in HCC cells, we further performed cell apoptosis assays when Akt was silenced by a validated siRNA (data not shown) or blocked by LY294002, a known inhibitor of PI3K-Akt signaling pathway. Interestingly, the results demonstrated that both siRNA against Akt and LY294002 inhibitor significantly abolished or even prevented the anti-apoptotic effect of FAM9C in Huh-7 and BEL-7404 cells with UV exposure $(\mathrm{P}<0.01)$ (Fig. 6D and F). Thus, these collective data suggest that FAM9C plays the anti-apoptotic role through mediating PI3K-Akt signaling pathway in $\mathrm{HCC}$ cells.

\section{Discussion}

FAM9 gene family mapped to Xp22.33-p22.31 includes three members, FAM9A, FAM9B and FAM9C, which are expressed 
A

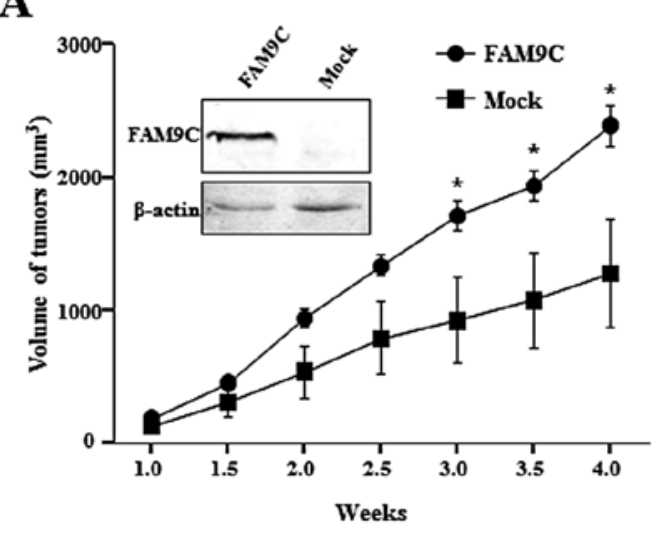

B

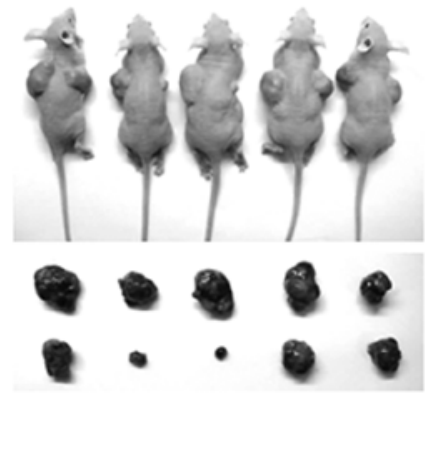

C

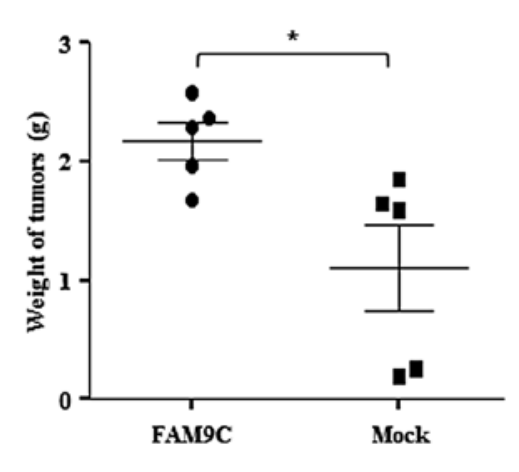

D

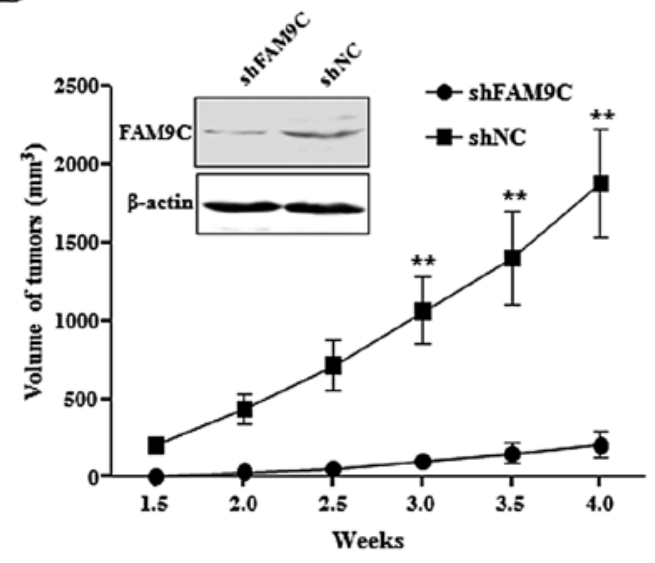

E

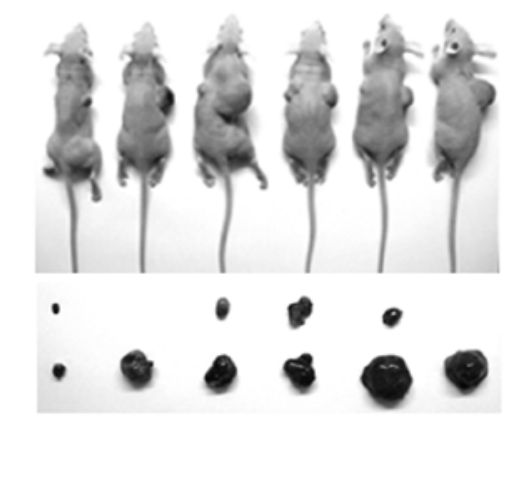

F

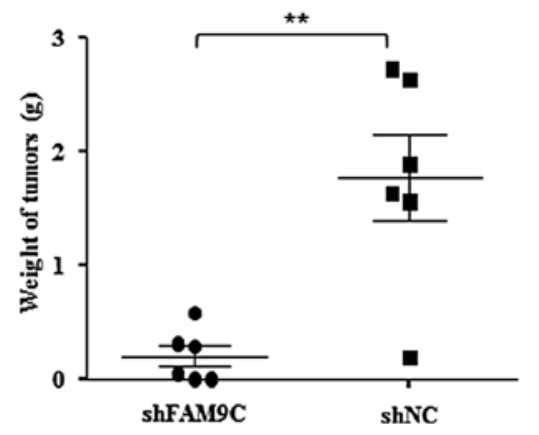

Figure 5. The effect of FAM9C on the tumorigenicity of HCC cells in nude mice. (A-C) Overexpressed FAM9C significantly promoted the tumorigenicity of Huh-7 cells in a flank of nude mice $(n=5)$, where the cells used as mock controls were injected s.c. into the opposite flank of the same mice. Tumor size was monitored at 0.5-week interval by using calipers (means \pm SEM) (A), then the removed tumors were photographed (B) and weighed (C). (D-F) Attenuated tumorigenicity of BEL-7404 cells in athymic nude mice $(n=6)$ endogenous FAM9C was stably silenced, where tumor growth kinetics was monitored at 0.5 -week interval (means \pm SEM) (D). The removed tumors were photographed (E) and weighted (F). A two-tailed t-test was used to evaluate the statistical significance of these experiments, as compared to the control. ${ }^{*} \mathrm{P}<0.05,{ }^{* *} \mathrm{P}<0.01$.

exclusively in testis (12) and have potential as cancer testis genes. In the present study, we first investigated their expression profiling of the three FAM9 family members in human HCC specimens. Notably, only FAM9C was frequently elevated in HCC specimens, suggesting that FAM9C could be a novel cancer testis gene. FAM9C expression was reactivated in HCC cells treated with the demethylating agent 5-aza-2'-deoxycitidine, implying that promoter demethylation was related to FAM9C deregulation in HCC. Although global DNA hypomethylation as a characteristic biomarker of liver cancer (15) has been identified as a cause of oncogenesis (16), some specific mechanisms especially the involvement in reactivated cancer testis genes are still obscure.

The crucial question is whether FAM9C upregulation contributes to neoplastic phenotype of HCC. As previously reported, restricted tissue-specific expression of FAM9C to testis, subcellular localization of the encoded protein to nucleus, and homology to SYCP3, encoding an essential structural component of the synaptonemal complex which is involved in synapsis, recombination and segregation of meiotic chromosomes, indicate FAM9C protein may be involved in the meiotic process (12). SYCP3 has been reported to be aberrantly expressed in tumors (17-19). SYCP3 impairs mitotic recombination by interfering with BRCA2, highlighting a new mechanism for chromosomal instability in cancers (20). However, the function of FAM9C gene is not yet determined by experiments. In the present study, FAM9C overexpression promoted cell proliferation, in vitro anchorage-dependent colony formation and in vivo tumorigenicity of Huh-7 cells. On the contrary, FAM9C knockdown induced by RNA interference inhibited cell proliferation, in vitro clonogenicity and in vivo tumorigenicity of BEL-7404 cells. These data showed that FAM9C as a novel cancer testis gene contributed to HCC and thereby could be a potential therapeutic target for HCC. However, further cell cycle analysis by flow cytometry showed FAM9C had no significant effect on cell cycle progression. Thus, whether FAM9C is involved in the formation of the synaptonemal complex or DNA replication are worthy further attention.

The molecular mechanism involved in these effects of FAM9C on HCC cells in vitro and in vivo, indicated that FAM9C was able to regulate the activation of Akt signaling pathway. PI3K/Akt/mTOR signaling pathway is commonly deregulated in a variety of cancers due to genetic alternations and promotes cellular growth, proliferation, and survival (21-24). Therefore, PI3K/Akt/mTOR signaling pathway 
A

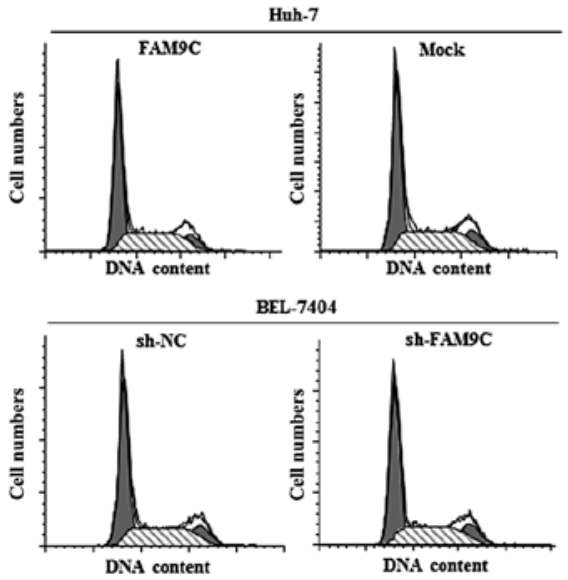

B
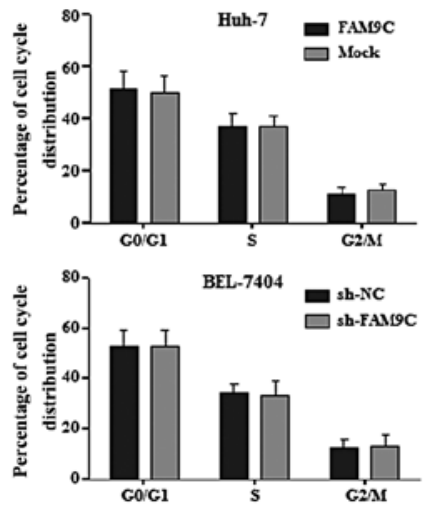

C



D
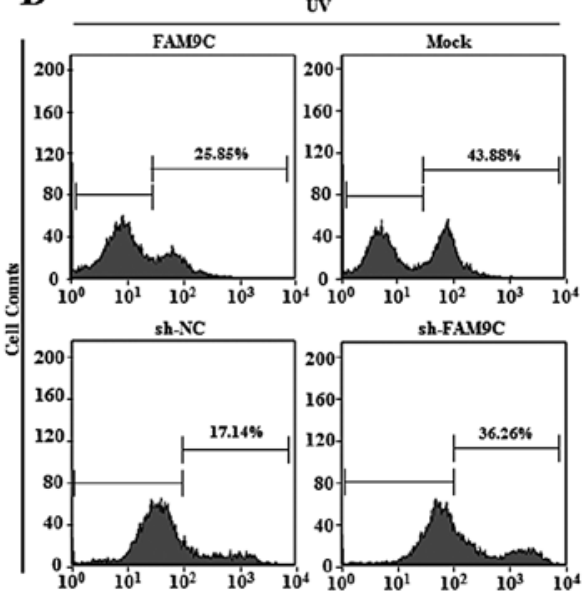

E

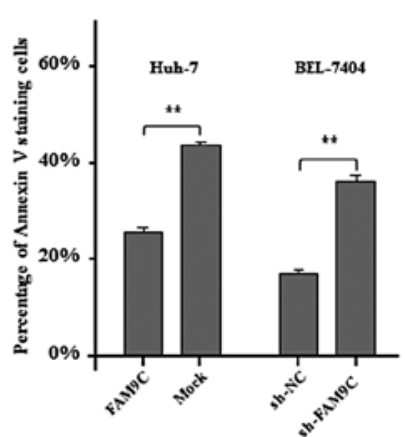

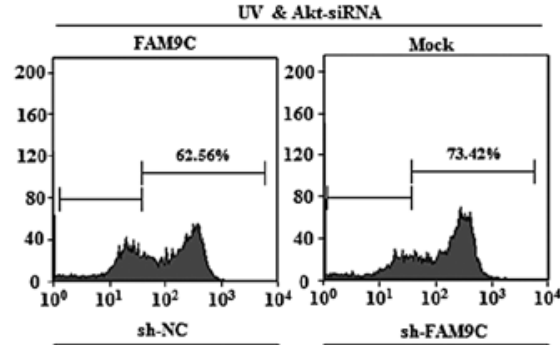

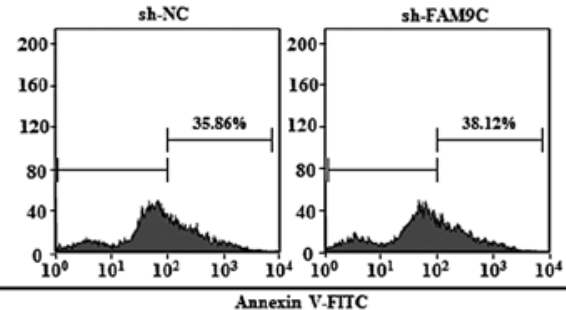

F
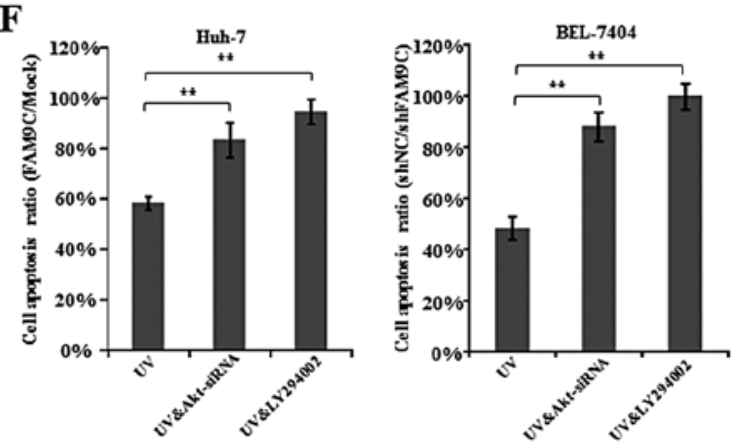

Figure 6. The anti-apoptotic role of FAM9C through regulating activation of Akt pathway in HCC cells. (A) Cell cycle analysis was performed by flow cytometry (FCM) and (B) the percentage of the cell population at different cell cycle phases was shown and statistically analyzed by a two-tailed t-test, in FAM9C overexpression in Huh-7 cells and FAM9C knockdown in BEL-7404 cells. (C) Western blot assay was employed to evaluate the phosphorylation level of Akt when FAM9C was overexpressed in Huh-7 cells and endogenous FAM9C was knocked down in BEL-7404 cells. (D) Cell apoptosis assay was performed by Annexin V-FITC staining using flow cytometry. The HCC cells were treated with UV exposure alone, or UV exposure plus Akt-siRNA, UV exposure plus LY294002, respectively. (E) The histogram represents the percentage of apoptotic cells from three FCM tests, FAM9C-expressing Huh-7 and FAM9Csilencing BEL-7404 cells were treated with UV exposure. (F) The results show the ratio of UV-induced apoptotic cell population as FAM9C overexpression in Huh-7 cells (left) and FAM9C knockdown in BEL-7404 cells (right) when Akt was silenced by a specific siRNA or was blocked by PI3K inhibitor LY294002. Statistical analysis was performed by a two-tailed t-test. ${ }^{*} \mathrm{P}<0.05,{ }^{* *} \mathrm{P}<0.01$.

represents a suitable and promising therapeutic target in $\mathrm{HCC}$, and many targeted agents against this signaling pathway are in clinical trials (25-28). In the present study, we revealed that upregulated FAM9C resulted in activation of Akt in HCC cells, implying that mutually targeting the FAM9C and PI3K/Akt/mTOR pathway may promote inhibitory effects on tumors. We have noted that the demethylating reagent,
5-Aza-2'-deoxycytidine (5-Aza-dC) exerted antitumor effect on HCC cells (29), but also caused the upregulation of FAM9C in $\mathrm{HCC}$ cells. In light of the FAM9C-promoting effects through the activation of Akt, we deduce that the combination of 5-Aza-dC and inhibitors of PI3K-Akt signaling pathway (e.g., LY294002) would more effectively inhibit proliferation of HCC cells. 
In conclusion, our findings demonstrated that FAM9C as a novel cancer testis gene played an anti-apoptotic role through regulating PI3K-Akt signaling pathway in human hepatocellular carcinoma. We propose that FAM9C represents a novel target for enhancing the response of HCC cells to demethylating reagents.

\section{Acknowledgements}

This research was supported in part by the Suzhou Administration of Science and Technology (SYS201046 and SZS201004).

\section{References}

1. Jemal A, Bray F, Center MM, Ferlay J, Ward E and Forman D: Global cancer statistics. CA Cancer J Clin 61: 69-90, 2011.

2. Siegel R, Naishadham D and Jemal A: Cancer statistics, 2013. CA Cancer J Clin 63: 11-30, 2013.

3. Kew MC: Epidemiology of hepatocellular carcinoma in subSaharan Africa. Ann Hepatol 12: 173-182, 2013.

4. Hoshida Y, Moeini A, Alsinet C, Kojima K and Villanueva A: Gene signatures in the management of hepatocellular carcinoma. Semin Oncol 39: 473-485, 2012.

5. Alsinet $\mathrm{C}$ and Villanueva $\mathrm{A}$ : Genomic prognostic markers in hepatocellular carcinoma. Gastroenterol Hepatol 35: 94-101, 2012 (In Spanish).

6. Sherman M: Modern approach to hepatocellular carcinoma. Curr Gastroenterol Rep 13: 49-55, 2011.

7. Simpson AJ, Caballero OL, Jungbluth A, Chen YT and Old LJ: Cancer/testis antigens, gametogenesis and cancer. Nat Rev Cancer 5: 615-625, 2005 .

8. Zhao L, Mou DC, Leng XS, et al: Expression of cancer-testis antigens in hepatocellular carcinoma. World J Gastroenterol 10: 2034-2038, 2004.

9. Peng JR, Chen HS, Mou DC, et al: Expression of cancer/testis (CT) antigens in Chinese hepatocellular carcinoma and its correlation with clinical parameters. Cancer Lett 219: 223-232, 2005.

10. Chen K, Huang W, Huang B, et al: BORIS, brother of the regulator of imprinted sites, is aberrantly expressed in hepatocellular carcinoma. Genet Test Mol Biomarkers 17: 160-165, 2013.

11. $\mathrm{Xu} \mathrm{H}, \mathrm{Gu} \mathrm{N}$, Liu ZB, et al: NY-ESO-1 expression in hepatocellular carcinoma: A potential new marker for early recurrence after surgery. Oncol Lett 3: 39-44, 2012.

12. Martinez-Garay I, Jablonka S, Sutajova M, Steuernagel P, Gal A and Kutsche K: A new gene family (FAM9) of low-copy repeats in Xp22.3 expressed exclusively in testis: implications for recombinations in this region. Genomics 80: 259-267, 2002.

13. Livak KJ and Schmittgen TD: Analysis of relative gene expression data using real-time quantitative PCR and the 2(-Delta Delta C(T)) method. Methods 25: 402-408, 2001
14. Kang TH, Noh KH, Kim JH, et al: Ectopic expression of X-linked lymphocyte-regulated protein $\mathrm{pM} 1$ renders tumor cells resistant to antitumor immunity. Cancer Res 70: 3062-3070, 2010.

15. Guerrero-Preston R, Santella RM, Blanco A, Desai M, Berdasco $M$ and Fraga M: Global DNA hypomethylation in liver cancer cases and controls: a phase I preclinical biomarker development study. Epigenetics 2: 223-226, 2007.

16. Akhavan-Niaki H and Samadani AA: DNA methylation and cancer development: molecular mechanism. Cell Biochem Biophys: Mar 19, 2013 (Epub ahead of print).

17. Niemeyer P, Tureci O, Eberle T, Graf N, Pfreundschuh M and Sahin U: Expression of serologically identified tumor antigens in acute leukemias. Leuk Res 27: 655-660, 2003.

18. Kalejs M, Ivanov A, Plakhins G, et al: Upregulation of meiosisspecific genes in lymphoma cell lines following genotoxic insult and induction of mitotic catastrophe. BMC Cancer 6: 6, 2006.

19. Mobasheri MB, Jahanzad I, Mohagheghi MA, Aarabi M, Farzan S and Modarressi MH: Expression of two testis-specific genes, TSGA10 and SYCP3, in different cancers regarding to their pathological features. Cancer Detect Prev 31: 296-302, 2007.

20. Hosoya N, Okajima M, Kinomura A, et al: Synaptonemal complex protein SYCP3 impairs mitotic recombination by interfering with BRCA2. EMBO Rep 13: 44-51, 2012.

21. De Luca A, Maiello MR, D'Alessio A, Pergameno $M$ and Normanno N: The RAS/RAF/MEK/ERK and the PI3K/AKT signalling pathways: role in cancer pathogenesis and implications for therapeutic approaches. Expert Opin Ther Targets 16 (Suppl 2): S17-S27, 2012.

22. Zhou Q, Wong CH, Lau CP, et al: Enhanced antitumor activity with combining effect of mTOR inhibition and microtubule stabilization in hepatocellular carcinoma. Int J Hepatol 2013: 103830, 2013.

23. Touil Y, Zuliani T, Wolowczuk I, et al: The PI3K/AKT signaling pathway controls the quiescence of the low-Rhodamine123retention cell compartment enriched for melanoma stem cell activity. Stem Cells 31: 641-651, 2012.

24. Almhanna K, Strosberg J and Malafa M: Targeting AKT protein kinase in gastric cancer. Anticancer Res 31: 4387-4392, 2011.

25. Gedaly R, Angulo P, Chen C, et al: The role of PI3K/mTOR inhibition in combination with sorafenib in hepatocellular carcinoma treatment. Anticancer Res 32: 2531-2536, 2012.

26. Muntane J, De la Rosa AJ, Docobo F, Garcia-Carbonero R and Padillo FJ: Targeting tyrosine kinase receptors in hepatocellular carcinoma. Curr Cancer Drug Targets 13: 300-312, 2013.

27. Temirak A, Abdulla $\mathrm{M}$ and Elhefnawi M: Rational drug design for identifying novel multi-target inhibitors for hepatocellular carcinoma. Anticancer Agents Med Chem 12: 1088-1097, 2012.

28. Grabinski N, Ewald F, Hofmann BT, et al: Combined targeting of AKT and mTOR synergistically inhibits proliferation of hepatocellular carcinoma cells. Mol Cancer 11: 85, 2012.

29. Tao SF, Zhang CS, Guo XL, et al: Anti-tumor effect of 5-aza-2'-deoxycytidine by inhibiting telomerase activity in hepatocellular carcinoma cells. World J Gastroenterol 18: 2334-2343, 2012. 